(6) OPEN ACCESS

'Department of Ophthalmology, University Hospital Bonn, Bonn, Germany

${ }^{2}$ Moorfields Eye Hospital NHS Foundation Trust, London, UK ${ }^{3}$ Institute of Ophthalmology, University College London, London, UK

${ }^{4}$ Oxford Eye Hospital, Oxford University Hospitals NHS Foundation Trust, and Nuffield Laboratory of Ophthalmology, Nuffield Department of Clinical Neurosciences, University of Oxford, Oxford, UK

\section{Correspondence to}

Mrs Simone Tzaridis,

Department of Ophthalmology, University of Bonn, Bonn 53127 Germany; Simone.Mueller@ukb. uni-bonn.de

Received 10 October 2018 Revised 14 December 2018 Accepted 5 February 2019

D Check for updates

(c) Author(s) (or their employer(s)) 2019. Re-use permitted under CC BY-NC. No commercial re-use. See rights and permissions. Published by BMJ.

To cite: Tzaridis $\mathrm{S}$, Heeren $\mathrm{T}$, Mai C, et al. Br J Ophthalmol Epub ahead of print: [please include Day Month Year]. doi:10.1136/

bjophthalmol-2018-313364

\title{
Right-angled vessels in macular telangiectasia type 2
}

\author{
Simone Tzaridis, ${ }^{1}$ Tjebo Heeren, ${ }^{\circledR 2,3}$ Clarissa Mai, ${ }^{1}$ Sarah Thiele, ${ }^{1}$ Frank G Holz, \\ Peter Charbel Issa, ${ }^{4}$ Philipp Herrmann ${ }^{1}$
}

\section{ABSTRACT \\ Purpose To evaluate the role of right-angled vessels (RAVs) during disease progression in macular telangiectasia type 2 (MacTel).}

Methods In this study, 100 eyes of 52 patients and 52 eyes of 26 age-related controls were examined using fundus photography, spectral-domain optical coherence tomography (SD-OCT), OCT angiography (OCT-A) and fundus fluorescein angiography (FFA). Two masked readers graded fundus photographs of patients' eyes into five disease stages according to Gass and Blodi, and evaluated all eyes for the presence of RAVs. If RAVs were present, their course and origin (arterial vs venous) was evaluated with OCT-A and FFA, respectively. Additionally, we looked for morphological correlates of these vessels on SD-OCT scans. Neovascular eyes were analysed for the presence of RAVs and for morphological changes on formation of neovascularisations (NVs).

Results In OCT-A, RAVs were already detectable in eyes with early stages (1 to 2), could be tracked from superficial to outer retinal layers and were shown to form anastomoses in the outer retina with disease progression. These vessels were of both arterial and venous origin as shown by early phase FFA. Dilated capillaries and RAVs in OCT-A corresponded to hyperreflective alterations of the outer retina on SD-OCT scans. In 19/19 eyes, NVs were associated with the presence of RAVs, and RAVs were shown to directly connect to neovascular complexes and to undergo morphological changes upon NV formation.

Conclusions The results emphasise the role of RAVs during disease progression from an early stage on and demonstrate their involvement in the development of secondary NVs in MacTel.

\section{INTRODUCTION}

Macular telangiectasia (MacTel) type 2 is a bilateral macular disease that is characterised by both neurodegenerative and vascular alterations, and a slowly progressive disease course. ${ }^{1}$ Structural and functional alterations are usually limited to an oval-shaped area centred on the fovea, of approximately $8^{\circ}$ horizontal and $5^{\circ}$ vertical diameter. In 1993, Gass and Blodi suggested five stages based on funduscopic and angiographic findings ${ }^{2}$ : stage 1 is characterised by the lack of typical funduscopic findings, but the presence of leakage in fluorescein angiography; stage 2 is defined by a loss of retinal transparency (retinal greying) and stage 3 by the occurrence of prominent, blunted retinal vessels that do not seem to narrow towards the foveola, but to dive into deeper retinal layers at a right angle ('right-angled vessels', RAVs). These vessels were described to occur either in a solitary or multiple form, and to frequently be associated with ectatic capillaries. ${ }^{1}$ Stage 4 is defined by the occurrence of intraretinal pigment migration and stage 5 by the presence of secondary neovascularisations (NVs).

Secondary NVs are considered a rare but vision-threatening complication in MacTel. ${ }^{1}{ }^{2}$ Their origin still remains controversial. Thus far, neovascular complexes were commonly thought to originate rather from the retinal vasculature than from the choroid, developing retinal-retinal anastomoses or chorioretinal anastomoses. ${ }^{2-4}$ Different studies observed morphological vascular changes preceding NV development, for example, dilation, elongation or apparent discontinuation of vesselsand especially of RAVs-as well as the occurrence of concomitant pigment plaques. ${ }^{235}$

Until recently, available imaging methods enabled only a two-dimensional illustration of the retinal/ choroidal vasculature. An en face differentiation into superficial and deep retinal plexus and exact allocation of vessels to different retinal layers has so far not been feasible. With the advent of optical coherence tomography angiography (OCT-A), the awareness of this has markedly increased. Showing motion contrast in the retina, OCT-A can provide detailed three-dimensional images of the perfused microvasculature in different retinal layers and in the choroid. ${ }^{6-11}$ In this study, we evaluate the role of microvascular changes and especially of RAVs during disease progression and NV development and take advantage of currently available imaging methods for a precise illustration of the retinal microvasculature in eyes with MacTel.

\section{METHODS}

In this cross-sectional analysis, participants were selected from the Natural History and Observation Study from a single-centre cohort at the Department of Ophthalmology, University Hospital of Bonn, Germany. Protocol details of the study have been published previously. ${ }^{12}$ The diagnosis of MacTel type 2 was based on funduscopy, spectral-domain OCT (SD-OCT), macular pigment optical density and fundus fluorescein angiography (FFA). ${ }^{1}$ Controls were age-matched healthy probands without any evidence of retinal diseases and clear media. Patients and controls underwent a full clinical examination, including best-corrected visual acuity testing, dilated funduscopy, SD-OCT (volume scans of $15 \mathrm{~mm} \times 10 \mathrm{~mm}$ [high-resolution mode, 97 scans], Spectralis; Heidelberg Engineering, Heidelberg, Germany) and OCT-A (scans of $3 \mathrm{~mm} \times 3 \mathrm{~mm}[312$ horizontal A-scans, Angio mode], Zeiss PLEX Elite 9000; Carl Zeiss Meditec, Dublin, California, USA). Additionally, fluorescein 
angiography (SLO; Heidelberg Engineering) was performed in patients only. Inclusion criteria were a complete imaging data set and sufficient imaging quality.

Images of patients' eyes prior to the development of secondary NVs were analysed, if available. All imaging modalities showing the morphology of the macular vasculature (including FFA, fundus autofluorescence [FAF], infrared imaging) were therefore included.

\section{Image processing and analysis}

For segmentation of the images (OCT-A and SD-OCT) into superficial, deep and avascular layers, and into choriocapillaris and outer retina-choriocapillaris layer (ORCC), a semiautomated segmentation algorithm was applied. Subsequently, segmentation lines were manually adjusted considering individual layer variation and shift in the context of the disease. ${ }^{13}$ Segmentation layers were based on relevant anatomical structures: the superficial layer was defined reaching from the outer boundary of the retinal nerve fibre layer to the outer boundary of the inner plexiform layer (IPL), the deep layer reaching from the outer boundary of IPL to the outer boundary of the outer plexiform layer (OPL) and the avascular layer reaching from the outer boundary of OPL to the outer boundary of the retinal pigment epithelium (RPE). The latter layer usually does not contain vascular networks in healthy subjects. The CC layer was defined as a slab reaching from the outer boundary of Bruch's membrane to approximately $20 \mu \mathrm{m}$ below Bruch's membrane. Additionally, for a good visualisation of NV membranes, the ORCC slab extending from the outer boundary of the OPL to 8 $\mu \mathrm{m}$ below Bruch's membrane was analysed.

After segmentation, image data were processed by a general sliding slab method in order to reduce decorrelation tail artefacts from superimposing structures. This method was previously described elsewhere. ${ }^{14} 15$

\section{Image grading}

Patients' eyes were graded according to the staging system proposed by Gass and Blodi ${ }^{2}$ based on the evaluation of fundus photographs and FFA. Furthermore, all eyes were analysed for the presence or absence of RAVs in fundus photography and OCT-A.

RAVs were defined as follows: in funduscopy/fundus photography visible, blunted, slightly dilated vessels that are mainly located in the temporal parafovea, and that seem not to narrow towards the foveola, but suddenly dive at a right angle into deeper retinal layers (definition according to the morphological criteria described by Gass and Blodi). ${ }^{12}$ In OCT-A, vessels bearing the same characteristics (dilation and apparent discontinuation caused by a vertical deviation from superficial to deeper retinal layers) were likewise defined as RAVs.

Vessels that met the criteria of RAVs in OCT-A were additionally grouped according to their venous or arterial origin based on early phase FFA.

SD-OCT images were graded for the presence or absence of hyper-reflective lesions located in the outer retina, and images were correlated to OCT-A data. Grading was performed in a masked fashion by two independent graders (STz and $\mathrm{CM}$ ). In case of disagreement between graders, a third grader (STh) performed the final grading.

\section{Statistical analyses}

Statistical analyses were performed using GraphPad Prism V.7.0 (GraphPad Software, San Diego, California, USA). Interobserver agreement between graders 1 and 2 was calculated using Cohen's kappa (к) test.

\section{RESULTS}

Data of 100 eyes of 52 patients (mean age of 62.3 years \pm SD 6.3 ; range, 49-78) and 52 eyes of 26 age-related controls (mean age of 61.2 years \pm SD 5.9; range, 45-75) were analysed. Four eyes were excluded from our analysis due to insufficient OCT-A image quality. Image grading revealed 16 stage 1, 13 stage 2, 29 stage 3, 23 stage 4 and 19 stage 5 eyes.

\section{Detection and imaging of RAVs}

According to the staging system of Gass and Blodi, RAVs are funduscopically not visible in early disease stages 1 and 2 . However, OCT-A imaging detected dilated, vertically running vessels that were defined as RAVs (see Methods section) in $11 / 16(69 \%)$ patients' eyes assigned to stage 1 , and in $10 / 13$ (77\%) patients' eyes assigned to stage 2 . These vessels were shown to dive into deeper retinal layers and form anastomoses that could be visualised in the deep and/or avascular layer by OCT-A imaging (figure 1). Notably, the extent of abnormal vessel formation in the avascular layer seemed to increase with disease progression (figure 2). Further, an association of RAVs with a rarefication of surrounding vessels in the deep plexus was observed in patients' eyes and seemed to increase with disease progression (figures 1-3). In eyes with advanced disease stages, enlarged intervascular spaces and rarefied vessels surrounding RAVs could also be detected in the superficial plexus. In none of the control eyes, similar vascular changes were observed.

A subgroup analysis of eyes with a largely preserved retinal architecture and thus well-definable retinal layers $(n=19$, stages 1 to 3 ; exemplary eyes are shown in figure 2, four upper rows) likewise revealed the presence of pathological vascular structures in the deep and avascular layers. Notably, in four eyes (all stage 1 ), vascular alterations were detectable in the superficial and deep layers, but did not seem to infiltrate the avascular layer (two out of these eyes are shown in the two upper rows of figure 2).

Interestingly, we observed an irregular appearance of vascular structures and an apparent increase of flow voids in the choriocapillaris of eyes with advanced disease stages (figures 2 and 3).

Comparison of OCT-A and conventional SD-OCT data revealed a high correlation of these dilated capillaries (and especially RAVs) in OCT-A to hyper-reflective intraretinal lesions at the level of the deep retinal plexus and the avascular layer (figure 2). Both alterations (in SD-OCT and OCT-A) were detectable in all disease stages and showed a parallel enlargement and extension to deeper layers/the avascular layer with disease progression. The degree of these changes, however, varied strongly between eyes. In eyes with early disease stages (see figure 2, four upper rows), we observed only scattered dilated capillaries in OCT-A that correlated to small, solitary spot-like hyper-reflective lesions on SD-OCT scans. In eyes with advanced disease stages, distinctly dilated vessels and their courses from inner to outer retinal layers could be directly correlated to large hyper-reflective intraretinal lesions that extended over several layers (figure 2, four lower rows).

The occurrence of concomitant funduscopically visible pigment plaques (in stages $\geq 4$ ) increased the intensity of the signal as well as the retrograde signal loss beyond the lesions causing pronounced shadowing effects on underlying structures. Control eyes showed neither hyper-reflective signalling beyond 


\section{Clinical science}
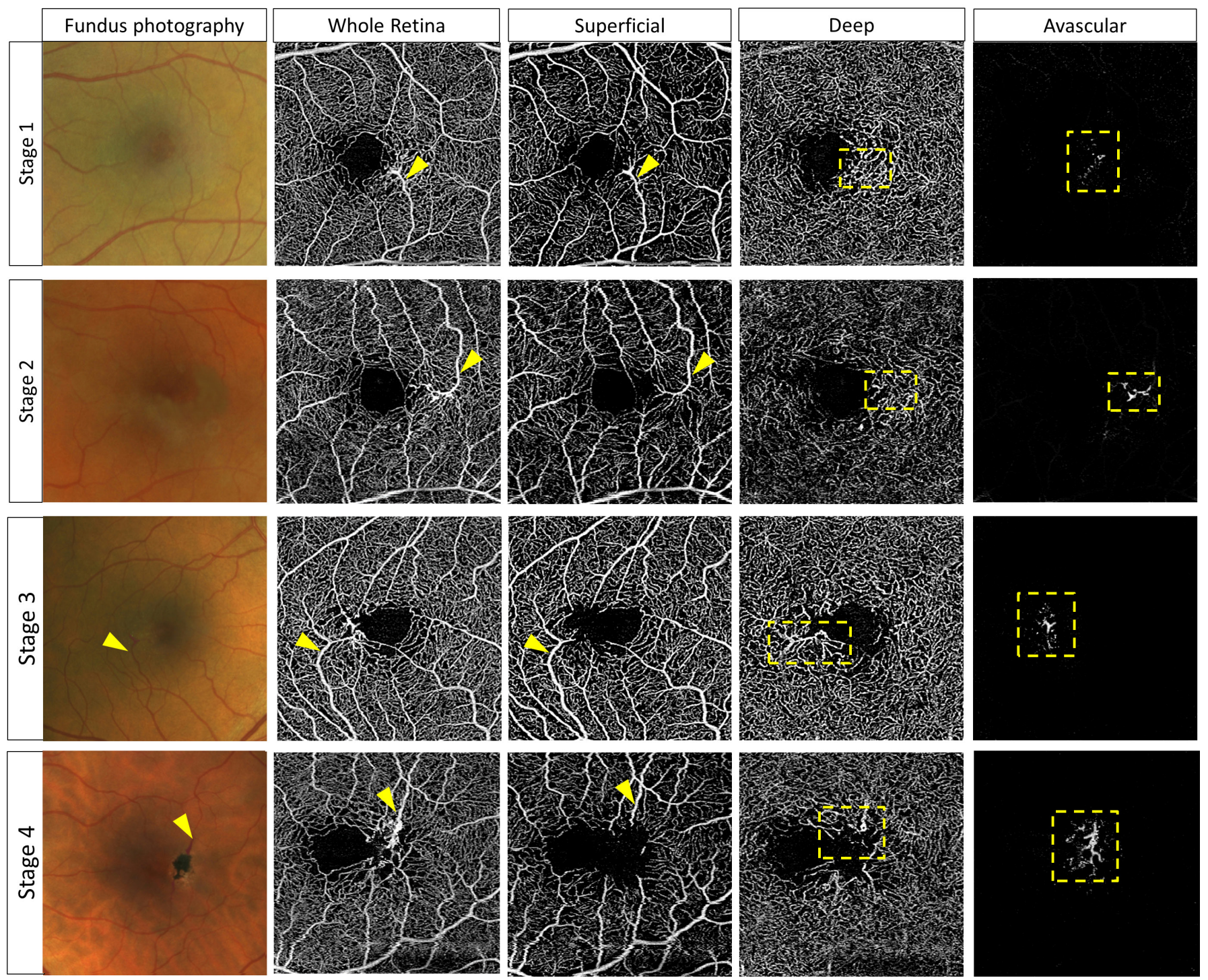

Figure 1 Right-angled vessels (RAVs) are present in early disease stages as shown by optical coherence tomography angiography (OCT-A) before they are visible in funduscopy. Fundus photographs and OCT-A (whole retina, superficial, deep and avascular layer) of exemplary eyes of different disease stages. RAVs and their courses are marked by yellow arrowheads. Anastomoses are marked with a yellow-dotted box in the deep and avascular layer, respectively. Note the increasing rarefication of vessels surrounding RAVs with disease progression in the deep and superficial layers.

the level of the OPL in SD-OCT, nor dilated capillaries/RAVs in OCT-A.

Analyses of FFA images showed that in all eyes (non-neovascular and neovascular) with available FFA (73/100), the observed anastomotic complexes in the outer retina were formed by vessels of both venous and arterial origin (figure 3). Notably, in most cases, these anastomoses were formed by vessels from the superior and inferior arcade, and usually consisted of pairs of at least one arterial and two venous vessels (figure 3).

\section{Secondary NVs in OCT-A}

In 19/19 (100\%) eyes that were classified as neovascular (neovascular membranes \pm haemorrhages, oedema), complexes of neovascular vessels were detectable in OCT-A (best visible in the ORCC layer; figure 4). In all neovascular eyes, RAVs were present and could be tracked from superficial to outer retinal layers in OCT-A. Notably, these vessels seemed to be directly connected to subretinal neovascular complexes, as shown in figure 4.
In cases where longitudinal data prior to NV development were available $(n=12)$, we observed these vessels to undergo morphological transformation with thickening, deformation (stretching, elongation, dilation) and apparent discontinuation with disease progression and NV formation (figure 5).

\section{Interobserver agreement}

The strength of interobserver agreement (between graders 1 and 2) was highest for the presence or absence of RAVs in OCT-A $(\kappa=0.83)$. Subdivision of eyes into disease stages 1 to 5 according to Gass and Blodi was best for advanced disease stages ( 3 to $5 ; \kappa=0.72$ ), and lower but still adequate for early stages ( 1 to $2 ; \kappa=0.62$ ).

\section{DISCUSSION}

According to the clinical staging system proposed by Gass and Blodi, the presence of so-called RAVs in funduscopy is considered a sign for a rather advanced disease form (stage 3 out of 


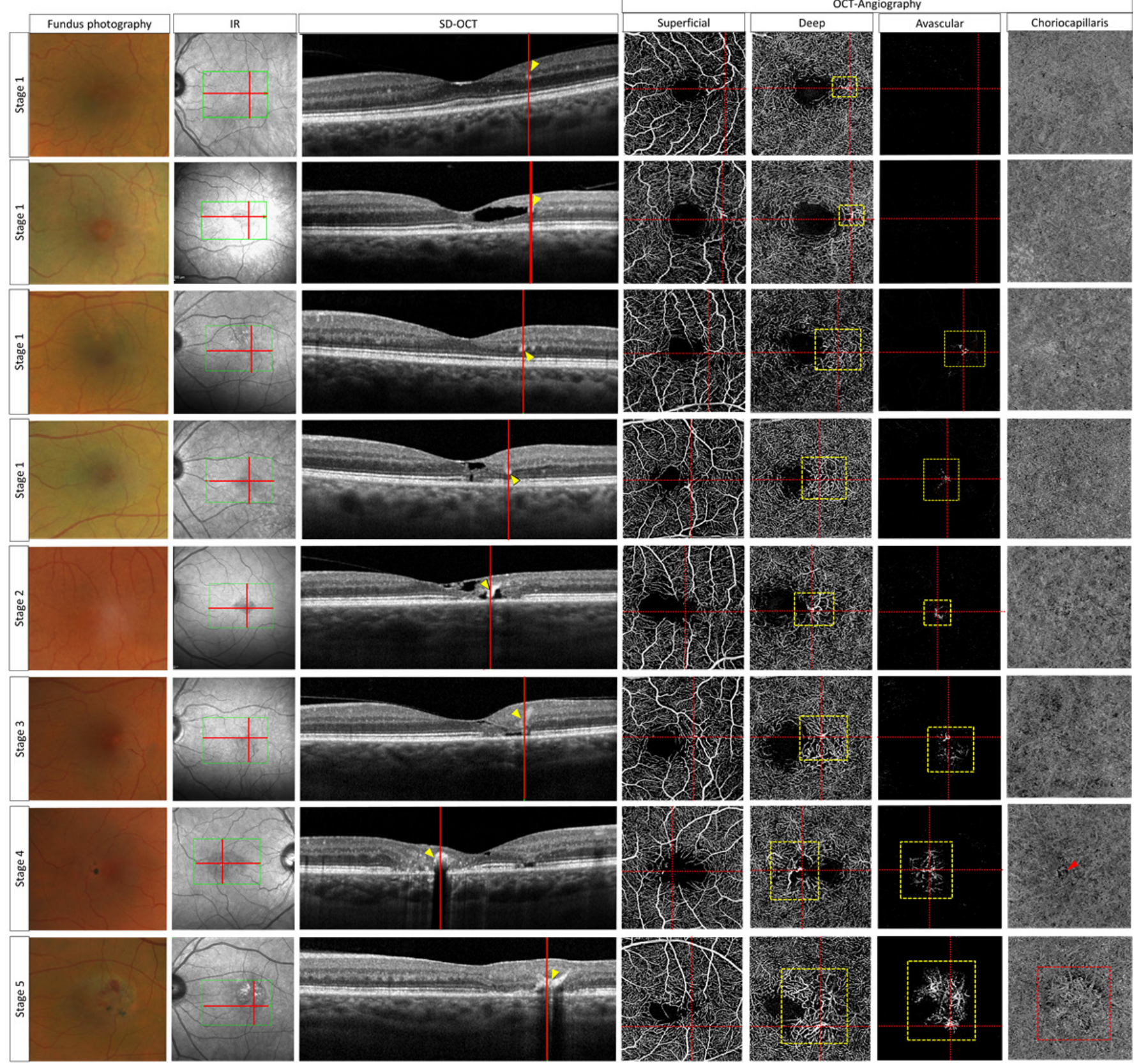

Figure 2 Intraretinal hyper-reflective lesions in the outer retina as observed in spectral-domain optical coherence tomography (SD-OCT) correspond to dilated capillaries and vertically running vessels (right-angled vessels, RAVs) in OCT angiography (OCT-A). These lesions are most pronounced in advanced, but may also be observed in early disease stages, and in eyes with a largely preserved retinal architecture (four upper rows). In the latter, prominent cut vessels could be visualised both in the deep and in the avascular layer in SD-OCT and OCT-A, respectively. Note that the intensity of the signal as well as the retrograde signal loss beyond the lesions increase with occurrence of concomitant pigment plaques (stages 4 and 5). Exemplary eyes out of different disease stages (stages 1 to 5 ) imaged with (from left to right) fundus photography, infrared (IR), SD-OCT and OCT-A (superficial, deep, avascular and choriocapillaris layer). The exact localisation of corresponding OCT -sections (longitudinal and transverse) is marked with red lines. Yellow arrowheads (SD-OCT) and dotted boxes (OCT-A) indicate abnormal vessels in the deep and in the avascular layer. A red-dotted box indicates the visible neovascular complex in the choriocapillaris layer of the stage 5 eye. The red arrowhead points out a large shadowing artefact in the choriocapillaris layer caused by an overlying pigment plaque in the stage 4 eye. Note the apparently increasing flow voids in the choriocapillaris layer in eyes with advanced disease stages.

5) in MacTel. $^{2}$ However, this classification is solely based on morphological findings as seen in funduscopy and FFA and does not consider findings from currently available imaging modalities such as OCT or OCT-A. In this study, we evaluated and illustrated the retinal microvasculature with multimodal imaging and focused on the role of RAVs in the context of disease progression in MacTel.
By means of OCT-A, we showed that vessels reflecting the morphological characteristics of RAVs could already be detected in early disease stages (stages 1 to 2 ) and before they became visible in funduscopy. Of course, other imaging modalities (eg, scanning laser ophthalmoscopy) with a higher contrast resolution and confocal lighting systems may also result in a better 

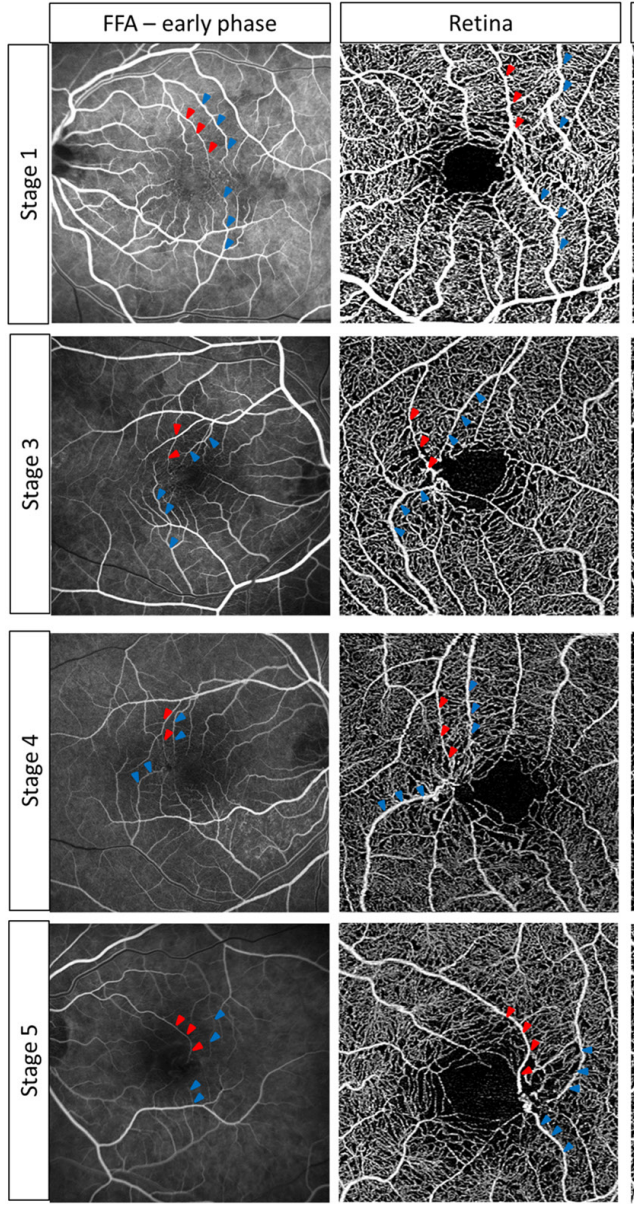
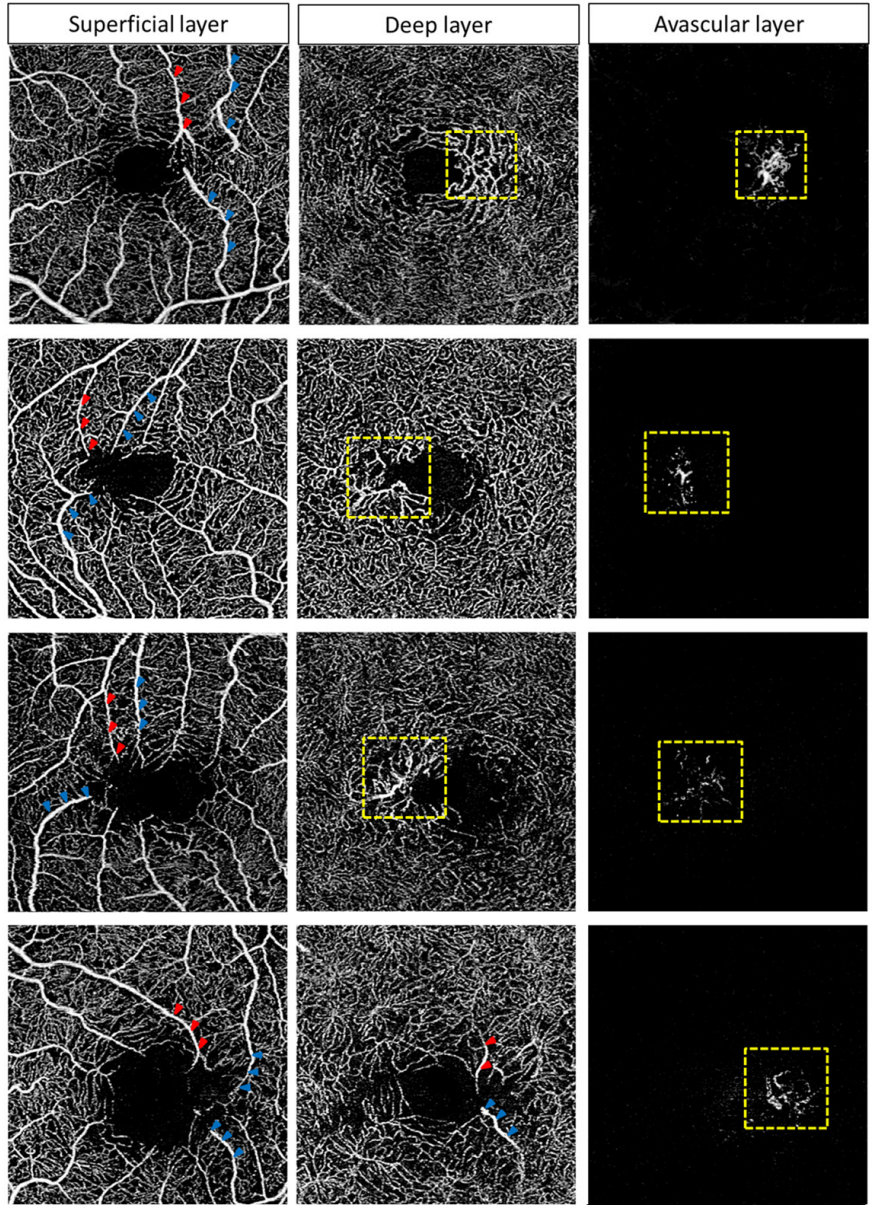
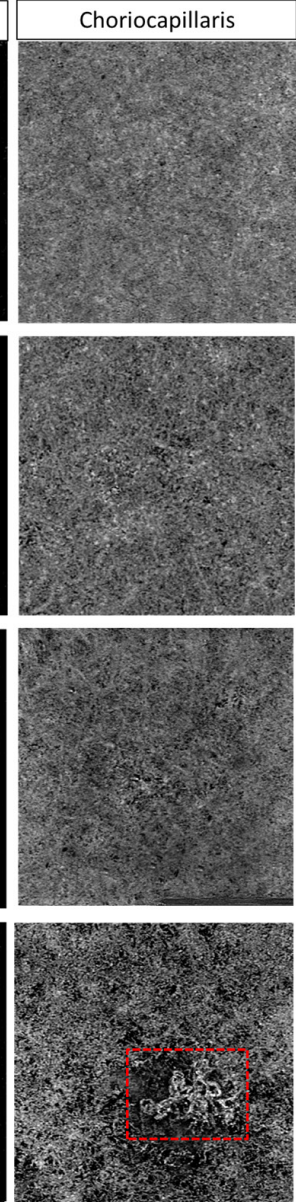

Figure 3 Right-angled vessels may be of venous and/ or arterial origin and may be tracked from superficial to deep retinal layers, forming anastomoses in the deep and/or avascular layer. Note the extension of vascular alterations to the outer retina (especially avascular layer) as well as the increasing rarefication of vessels with disease progression. Early phase fundus fluorescein angiography (FFA) and optical coherence tomography angiography (whole retina, superficial, deep, avascular and choriocapillaris layer) in exemplary eyes of different disease stages. Venous vessels are marked with blue, arterial vessels with red arrowheads. The red-dotted box indicates the visible neovascular complex in the choriocapillaris layer of the stage 5 eye.

visualisation and thus earlier detection of RAVs when compared with conventional funduscopy/fundus photography.

Our findings indicate that RAVs, and vascular alterations in general, may be affected earlier than previously expected.

Recent OCT-A studies in MacTel revealed vascular alterations, for example, a reduced vessel density, to possibly originate in the deep vascular plexus, and to further extend to the superficial plexus and the outer retina with disease progression. ${ }^{16-18}$ This is in accordance with our results showing an association of RAVs and a rarefication of surrounding vessels in the deep layer that seemed to increase with disease progression, and was also detectable in the superficial layer of eyes with advanced disease stages.

While alterations of the retinal vasculature have been described in detail, the role of vascular changes in the choriocapillaris and choroid in MacTel is still obscure. So far, the involvement of choroidal vessels in the context of secondary NVs in MacTel has been controversially discussed. ${ }^{4519}$ A potential affection of the choroidal vasculature in eyes with non-proliferative disease stages, however, has not been unravelled so far. In this study, we found increasing flow voids and irregularities in the choriocapillaris of eyes with advanced (but non-proliferative) disease stages. This finding, however, must be interpreted with caution as superimposing structures (eg, large vessels, pigment plaques) cause shadowing artefacts and may thus reinforce the impression of an altered choriocapillaris structure. Nevertheless, structural alterations of the choriocapillaris in eyes with non-proliferative disease stages are conceivable and might precede the development of secondary NVs. Further studies are warranted in order to verify and if applicable quantify these alterations and further assess underlying pathophysiological mechanisms.

The comparative analyses of OCT-A and SD-OCT data in this study revealed a direct correlation of abnormally dilated capillaries, and especially RAVs, in the deep retinal plexus as illustrated by OCT-A and hyper-reflective lesions in the outer retina as shown by SD-OCT. Both alterations were found to be present in all disease stages, and a gradual increase and extension to deeper retinal layers, including the avascular layer, was observed with disease progression. Our results are in line with previous observations of Baumüller et al, who first described the presence of hyper-reflective spots in the outer retina of eyes with MacTel and discussed these changes to possibly represent altered retinal capillaries. ${ }^{20}$ In the context of current and future clinical trials as well as in daily clinical practice, these findings might be helpful for a better judgement of the disease stage and progression.

So far, RAVs were believed to represent mainly dilated venules, which is probably true for most of those vessels, that we can observe in funduscopy. Yannuzzi et al, however, proposed some of these vessels to be arteriolar in nature and to form 

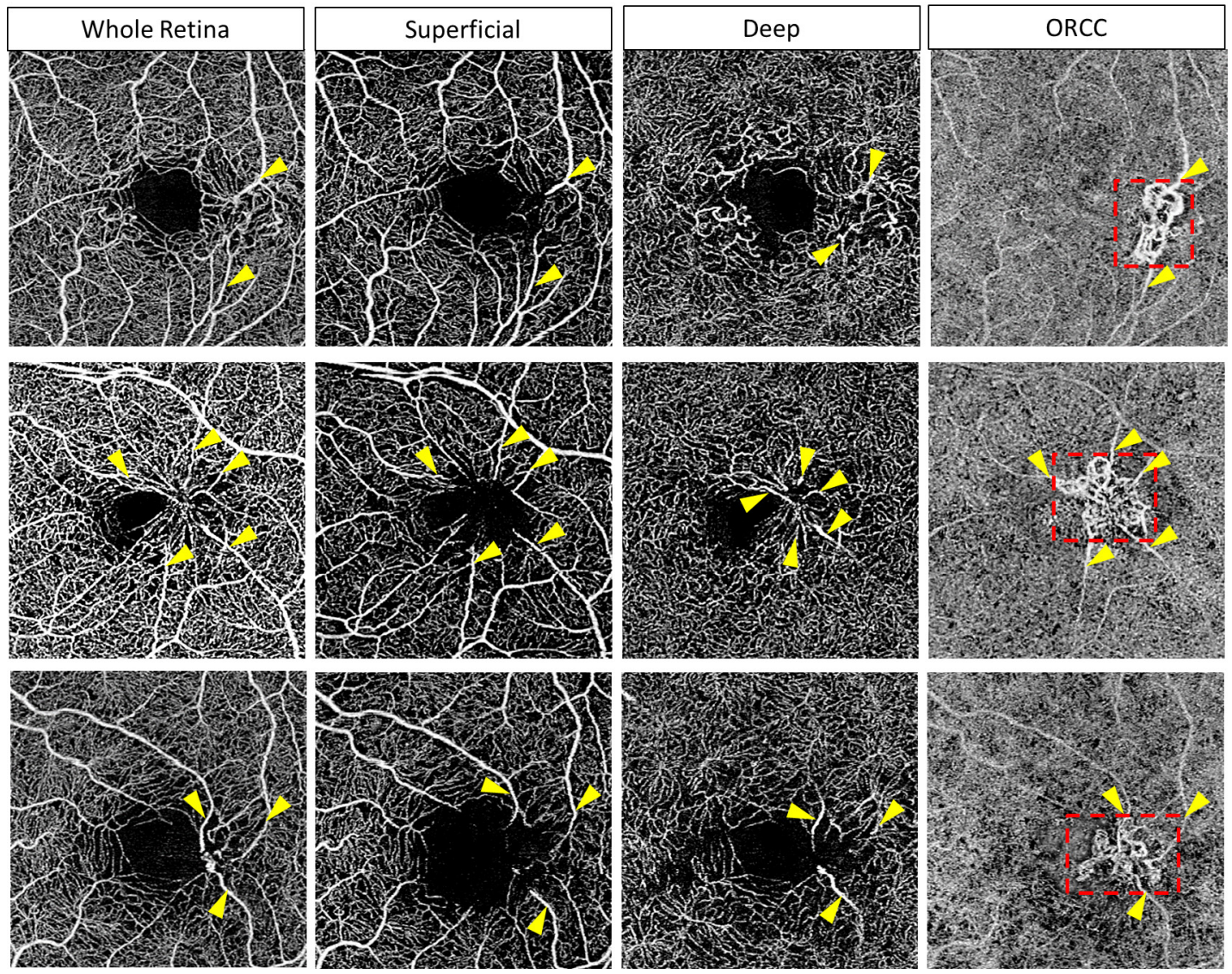

Figure 4 Right-angled vessels (marked with yellow arrowheads) directly connect to neovascular subretinal complexes (marked with a red-dotted box). Three exemplary eyes with secondary neovascularisation (NV) development in optical coherence tomography angiography (whole retina, superficial, deep and outer retina-choriocapillaris [ORCC] layers). NV complexes are best visible in ORCC.

retinal-retinal anastomoses. ${ }^{5}$ In accordance with these findings, we showed examples of arterial vessels with similar characteristics as RAVs (prominent, dilated, diving in a right angle into the deeper retina) by means of OCT-A and FAF. Also, we showed that anastomoses in the outer retina are connected and thus probably fed and drained by these superficially visible, bending vessels of arterial and venous origin. Interestingly, the formation of anastomoses and the extent of abnormal vessel formation in the avascular layer seemed to increase with disease progression. As we observed vascular structures in the avascular layer to occur even in eyes with a preserved retinal architecture (see figure 2, four upper rows), we concluded that this phenomenon was not solely simulated by a progressive collapse of retinal layers and increasing atrophy, but conceivably by a real downward growth or shift of pathological vessels to the outer retina. Findings from present OCT-A studies that proposed vascular changes to arise in the deep vascular plexus, and to extend to more superficial and deeper retinal layers with disease progression, ${ }^{16}{ }^{19}$ may further support our presumption of a gradual downgrowth of pathological vessels. Our findings are also in line with recently published data of Spaide et al, who observed a descent of vessels from the deep vascular plexus towards the RPE as well as the formation of retinal-retinal and retinal-choroidal anastomoses, that were shown to be highly associated with the presence of RAVs in eyes with MacTel. ${ }^{21}$

The occurrence of secondary NVs in MacTel may overall be considered a rather rare but serious complication, as it may result in a rapid loss of visual acuity. ${ }^{12}$ According to current knowledge, NVs may occur in every stage of the disease. ${ }^{1}$ Particular risk factors and the underlying pathomechanisms have not been unravelled so far. In this study, we showed an association of RAVs and NVs: In all 19 eyes with neovascular disease stage, prominent RAVs connecting the inner retina to the deep neovascular membranes were present. Furthermore, we observed morphological changes (dilation, stretching, apparent discontinuation etc) of these vessels to occur prior to or parallel with NV formation. This is in accordance with findings from previous studies that likewise found an altered morphology of retinal vessels preceding the development of NVs. ${ }^{235}$ Thus, growth of RAVs to the outer retina may be rated as a sign of disease progression. Morphological alterations of these vessels may suggest a high risk of NV formation and should be monitored closely.

\section{Study limitations}

The occurrence of atrophy and NV-associated changes resulting in an altered retinal architecture complicates the segmentation of OCT images and causes imaging artefacts that may influence the test results despite careful review and manual correction of segmentation. Furthermore, shadowing artefacts of large vessels and pigment plaques may impede the evaluation of subjacent structures (especially in the avascular and choriocapillaris layer), though an algorithm subtracting artefacts caused by superimposing structures was applied.

Our analyses in this study were limited to qualitative assessments of image data. Further studies are warranted in order to quantitatively assess different parameters and verify our observations in eyes with different disease stages. 

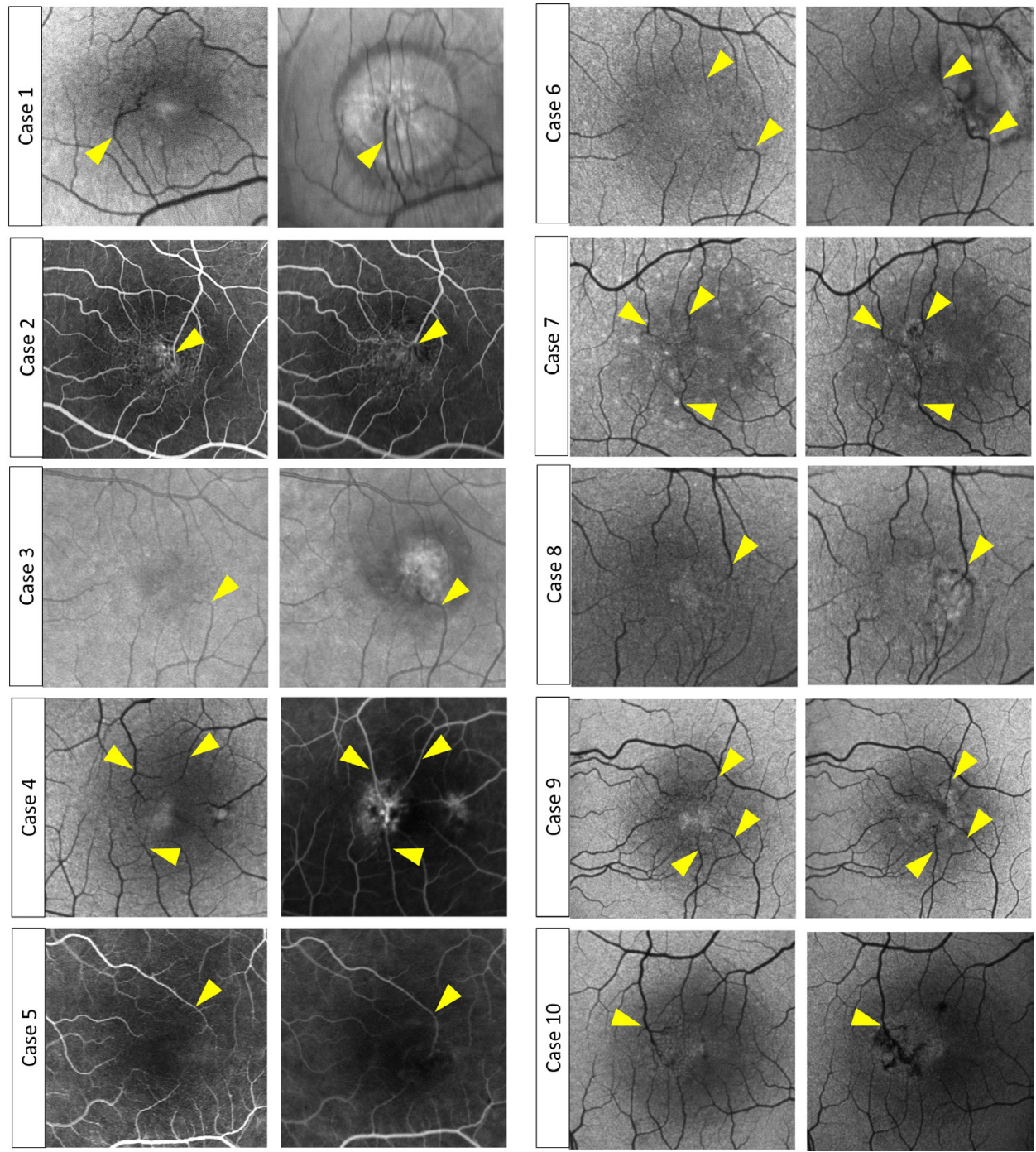

Figure 5 Exemplary illustration of morphological changes of right-angled vessels and accompanying vessels (marked with yellow arrowheads) during disease progression and development of secondary neovascularisations (NVs) (due to availability, different imaging modalities [fundus autofluorescence, fluorescein angiography, infrared] are illustrated). Each case shows the same eye prior to (left column) and after NV formation (right column). Morphological alterations include stretching and elongation, dilation and malformation, apparent discontinuation and increasing pigment accumulation.

Notably, in order to ensure an even distribution of eyes with early and advanced disease stages, our patient cohort was accordingly preselected. Thus, in our patient cohort, the distribution of disease stages does not reflect the normal distribution in MacTel, and early and neovascular disease stages are over-represented.

This study was conducted in a cross-sectional manner. Future collection of longitudinal data is needed in order to verify our results regarding a potential impact of RAVs on disease progression and $\mathrm{NV}$ formation.

\section{CONCLUSIONS}

In this study, we emphasise the role of RAVs during disease progression and their association with the development of secondary NVs in MacTel. Our results point out to a direct correlation of abnormally dilated capillaries in OCT-A and hyper-reflective lesions in the outer retina in SD-OCT. We contribute further knowledge about the direction of vessel growth and proliferation that may help to understand pathophysiological mechanisms of the disease and could function as a novel biomarker for disease and NV monitoring.
Contributors STz, STh, CM: data acquisition and evaluation. All authors: critical revision of the manuscript.

Funding This work was supported by the Lowy Medical Research Institute, La Jolla, USA; ProRetina, Bonn, Germany and the National Institute for Health Research (NIHR) Oxford Biomedical Research Centre (BRC), Oxford, UK.

Competing interests None declared.

Patient consent for publication Obtained.

Ethics approval The study was approved by the local ethics committee of the Friedrich-Wilhelms-University of Bonn, Bonn, Germany, and was conducted in accordance to the declaration of Helsinki.

Provenance and peer review Not commissioned; externally peer reviewed.

Open access This is an open access article distributed in accordance with the Creative Commons Attribution Non Commercial (CC BY-NC 4.0) license, which permits others to distribute, remix, adapt, build upon this work non-commercially, and license their derivative works on different terms, provided the original work is properly cited, appropriate credit is given, any changes made indicated, and the use is non-commercial. See: http://creativecommons.org/licenses/by-nc/4.0/.

\section{REFERENCES}

1 Charbel Issa P, Gillies MC, Chew EY, et al. Macular telangiectasia type 2. Prog Retin Eye Res 2013;34:49-77. 
2 Gass JD, Blodi BA. Idiopathic juxtafoveolar retinal telangiectasis. Update of classification and follow-up study. Ophthalmology 1993;100:1536-46.

3 Engelbrecht NE, Aaberg TM, Sung J, et al. Neovascular membranes associated with idiopathic juxtafoveolar telangiectasis. Arch Ophthalmol 2002;120:320-4.

4 Davidorf FH, Pressman MD, Chambers RB. Juxtafoveal telangiectasis - a name change? Retina 2004:24:474-8.

5 Yannuzzi LA, Bardal AMC, Freund KB, et al. Idiopathic macular telangiectasia. Arch Ophthalmol 2006:124:450-60.

6 Spaide RF, Fujimoto JG, Waheed NK. Optical coherence tomography angiography. Retina 2015;35:2161-2.

7 Choi W, Mohler KJ, Potsaid B, et al. Choriocapillaris and choroidal microvasculature imaging with ultrahigh speed OCT angiography. PLoS One 2013;8:e81499.

8 Kim DY, Fingler J, Zawadzki RJ, et al. Optical imaging of the chorioretinal vasculature in the living human eye. Proc Natl Acad Sci U S A 2013;110:14354-9.

9 Wang RK. Optical microangiography: a label free 3D imaging technology to visualize and quantify blood circulations within tissue beds in vivo. IEEE J Sel Top Quantum Electron 2010;16:545-54.

10 Wang RK, An L, Francis P, et al. Depth-resolved imaging of capillary networks in retina and choroid using ultrahigh sensitive optical microangiography. Opt Lett 2010;35:1467-9.

11 Wang RK, Jacques SL, Ma Z, et al. Three dimensional optical angiography. Opt Express 2007;15:4083-97.

12 Clemons TE, Gillies MC, Chew EY, et al. Baseline characteristics of participants in the natural history study of macular telangiectasia (MacTel) MacTel project report no. 2. Ophthalmic Epidemiol 2010;17:66-73.
13 Spaide RF, Curcio CA. Evaluation of segmentation of the superficial and deep vascular layers of the retina by optical coherence tomography angiography instruments in normal eyes. JAMA Ophthalmol 2017;135:259-62.

14 Bagherinia $\mathrm{H}$, Knighton RW, De Sisternes L, et al. A fast method to reduce Decorreltion tail artifacts in OCT angiography. Invest Ophthalmol Vis Sci 2017;58.

15 Wintergerst MWM, Pfau M, Müller PL, et al. Optical coherence tomography angiography in intermediate uveitis. Am J Ophthalmol 2018;194:35-45.

16 Chidambara L, Gadde SGK, Yadav NK, et al. Characteristics and quantification of vascular changes in macular telangiectasia type 2 on optical coherence tomography angiography. Br J Ophthalmol 2016;100:1482-8.

17 Spaide RF, Klancnik JM, Cooney MJ. Retinal vascular layers in macular telangiectasia type 2 imaged by optical coherence tomographic angiography. JAMA Ophthalmol 2015;133:66-73

18 Thorell MR, Zhang Q, Huang Y, et al. Swept-source OCT angiography of macular telangiectasia type 2. Ophthalmic Surg Lasers Imaging Retina 2014;45:369-80.

19 Zhang Q, Wang RK, Chen C-L, et al. Swept source optical coherence tomography angiography of neovascular macular telangiectasia type 2. Retina 2015;35:2285-99.

20 Baumüller S, Charbel Issa P, Scholl HPN, et al. Outer retinal hyperreflective spots on spectral-domain optical coherence tomography in macular telangiectasia type 2 . Ophthalmology 2010;117:2162-8.

21 Spaide RF, Yannuzzi LA, Maloca PM. Retinal-choroidal anastomosis in macular telangiectasia type 2. Retina 2018;38:1920-9. 